\title{
The Impact of Admission Serum Creatinine on Major Adverse Clinical Events in ST-Segment Elevation Myocardial Infarction Patients Undergoing Primary Percutaneous Coronary Intervention
}

\author{
Poornima Vinoda, Taylor Kann ${ }^{\mathrm{a}}$, Shyam Polaconda ${ }^{\mathrm{b}}$, Alibel Bello ${ }^{\mathrm{a}}$, Mohamed Khayata ${ }^{\mathrm{a}}$, \\ Fernando Munoz ${ }^{\mathrm{c}}$, Vinod Krishnappa ${ }^{\mathrm{b}}$, Rupesh Raina ${ }^{\mathrm{d}}$, e
}

\begin{abstract}
Background: Impaired renal function has been shown in previous studies to be an independent predictor of cardiovascular adverse events amongst patients admitted for percutaneous coronary intervention (PCI) following ST-segment elevation myocardial infarction (STEMI). This study investigates the impact of admission serum creatinine ( $\mathrm{SCr}$ ) on major cardiovascular outcomes among STEMI patients undergoing PCI.
\end{abstract}

Methods: A retrospective study of patients admitted for PCI following STEMI was conducted using the National Cardiovascular Database Action Registry (NCDR) at Cleveland Clinic Akron General (CCAG) Hospital. The primary outcome was a composite of major clinical events: cardiogenic shock, atrial fibrillation, ventricular tachycardia/fibrillation, heart failure, bleeding and mechanical ventilation. SCr was an independent and continuous variable.

Results: A total of 656 patients included in the study with the diagnosis of STEMI who subsequently underwent primary PCI. Patients with eGFR $<60 \mathrm{~mL} / \mathrm{min} / 1.73 \mathrm{~m}^{2}$ on admission had an increased incidence of cardiogenic shock $(\mathrm{P}=0.001)$, bleeding $(\mathrm{P}<0.001)$, heart failure $(\mathrm{P}<0.0005)$ and higher mortality rates $(\mathrm{P}=0.0005)$. Furthermore, in the setting of STEMI, elevated SCr was also associated with an increased risk of developing major adverse events like cardiogenic shock $(P=0.05)$, bleeding $(P=0.05)$, and heart failure

Manuscript submitted February 18, 2018, accepted April 10, 2018

aDepartment of Internal Medicine, Cleveland Clinic Akron General, Akron, $\mathrm{OH}$, USA

bAkron Nephrology Associates/Cleveland Clinic Akron General, Akron, OH, USA

'Department of Cardiology, Cleveland Clinic Akron General, Akron, OH, USA

dDepartment of Nephrology, Cleveland Clinic Akron General, Akron, OH, USA

${ }^{\mathrm{e} C o r r e s p o n d i n g ~ A u t h o r: ~ R u p e s h ~ R a i n a, ~ D e p a r t m e n t ~ o f ~ N e p h r o l o g y, ~ C l e v e-~}$ land Clinic Akron General and Akron Children's Hospital, Akron, $\mathrm{OH} 44308$, USA. Email: rraina@akronchildrens.org

doi: https://doi.org/10.14740/cr689w $(\mathrm{p}=0.005)$.

Conclusions: In the setting of STEMI, elevated SCr and eGFR $<60$ $\mathrm{mL} / \mathrm{min} / 1.73 \mathrm{~m}^{2}$ was associated with an increased risk of developing major adverse events including cardiogenic shock, bleeding and heart failure.

Keywords: ST-segment elevation myocardial infarction; Percutaneous coronary intervention; Serum creatinine; Glomerular filtration rate; Heart failure; Atrial fibrillation

\section{Introduction}

Impaired renal function has been established as a significant and independent predictor of adverse cardiovascular events among patients admitted for ST- segment elevation myocardial infarction (STEMI) receiving percutaneous coronary intervention (PCI) [1]. There have been limited studies that investigated the role of admission serum creatinine ( $\mathrm{SCr}$ ) on major adverse clinical events in patients with STEMI. Three recent studies have demonstrated a positive correlation between decreased eGFR and increased risk of atherosclerosis and reduced left ventricular ejection fraction (EF) in acute coronary syndrome [2-4]. These studies found that patients with eGFR $<60 \mathrm{~mL} / \mathrm{min} / 1.73 \mathrm{~m}^{2}$ had a 7.78 -fold higher mortality as compared to patients with an eGFR $>60 \mathrm{~mL} / \mathrm{min} / 1.73 \mathrm{~m}^{2}$ [24]. Here we investigate the impact of admission $\mathrm{SCr}$ on major adverse cardiovascular outcomes among STEMI patients undergoing PCI.

\section{Patients and Methods}

A retrospective study of patients, who were admitted for STEMI between January 2011 and September 2015 and have undergone primary PCI, was conducted using the National Cardiovascular Database Action Registry (NCDR) at Cleveland Clinic Akron General (CCAG) hospital. The study was 
Table 1. Demographics and Clinical Characteristics of Patients

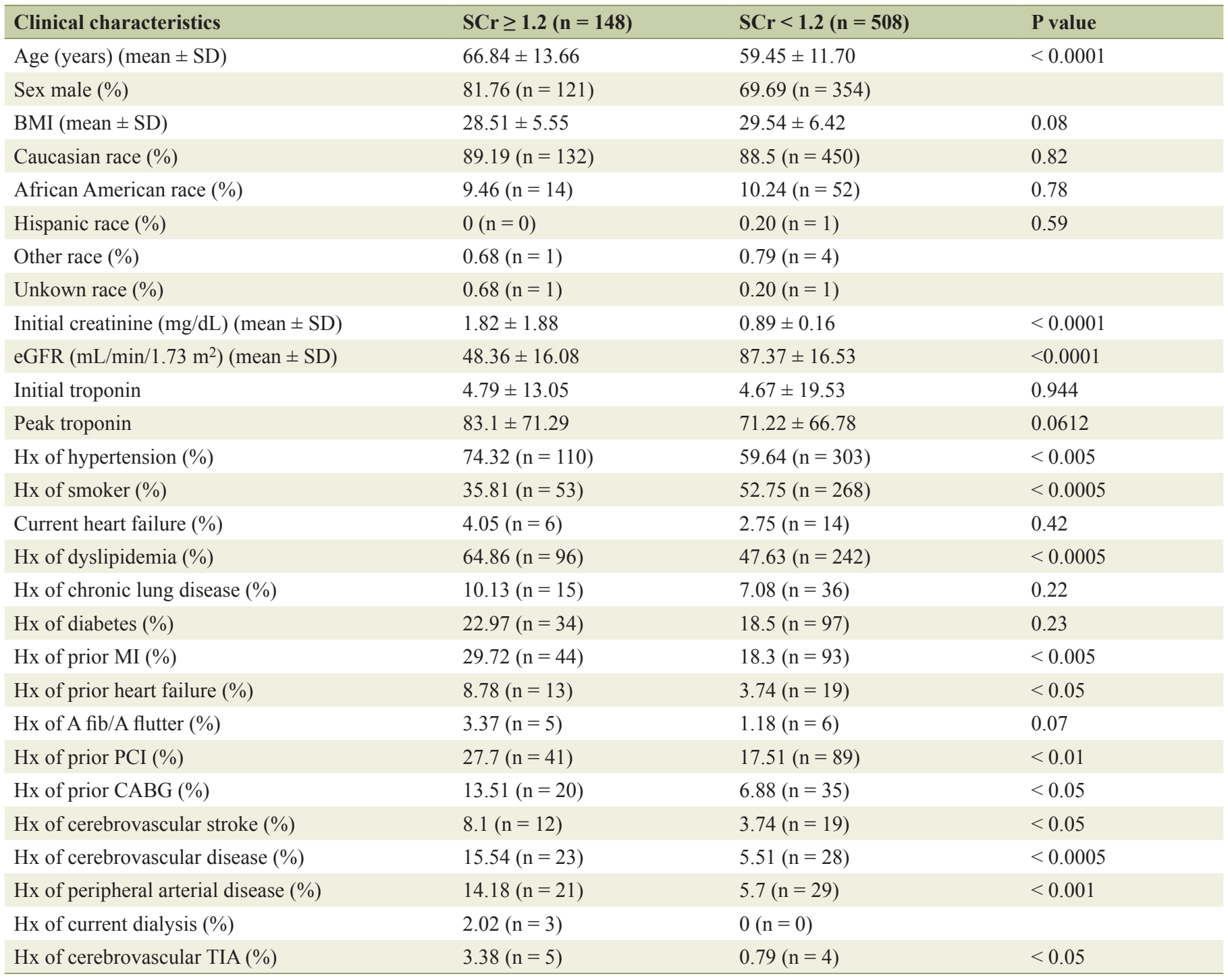

Scr: serum creatinine at admission; BMI: body mass index; eGFR: estimated glomerular filtration rate; Hx: history; MI: myocardial infarction; PCl: percutaneous coronary intervention; CABG: coronary artery bypass grafting; TIA: transient ischemic attack; A fib: atrial fibrillation; A flutter: atrial flutter.

approved by Institutional Review Board (IRB). The American College of Cardiology Foundation/American Heart Association (ACCF/AHA) guideline was used to define STEMI. Data elements include demographics, detailed medical history, medications, $\mathrm{SCr}$ at admission, and clinical outcomes during hospital stay. The primary outcome was a composite of major clinical events (cardiogenic shock, atrial fibrillation, ventricular fibrillation, ventricular tachycardia, heart failure, bleeding, and mechanical ventilation). Creatinine was the independent variable. Using below mentioned Chronic Kidney Disease Epidemiology Collaboration (CKD-EPI) equation, SCr (mg/ dL) based estimated glomerular filtration rate (eGFR) was calculated. Patient weight is not needed for CKD-EPI equation as the results are normalized to accepted average adult body surface area of $1.73 \mathrm{~m}^{2}$ and the use of this equation is advocated if eGFR is $>60 \mathrm{~mL} / \mathrm{min} / 1.73 \mathrm{~m}^{2}$ is desired [5].
$\mathrm{GFR}=141 \times \min \left(\mathrm{S}_{\mathrm{cr}} / \kappa, 1\right)^{\alpha} \times \max \left(\mathrm{S}_{\mathrm{cr}} / \kappa, 1\right)^{-1.209} \times 0.993^{\text {age }}$ $\times 1.018$ (if female) $\times 1.159$ (if black)

$\mathrm{S}_{\mathrm{cr}}$ : serum creatinine $(\mathrm{mg} / \mathrm{dL}) ; \kappa: 0.7$ for females and 0.9 for males; $\alpha$ : -0.329 for females and -0.411 for males; min: minimum of $\mathrm{S}_{\mathrm{cr}} / \kappa$ or 1 ; max: maximum of $\mathrm{S}_{\mathrm{cr}} / \kappa$ or 1

SPSS software was used to calculate descriptive statistics and perform multivariate analysis. Categorical variables were compared using chi-square test and the differences between the observed means in the two groups were calculated using two-sample $t$-test.

\section{Results}

A total of 656 patients admitted to CCAG hospital between January 2011 and September 2015 with the diagnosis of STE- 
Table 2. Adverse Clinical Outcomes Based on Admission Serum Creatinine and eGFR

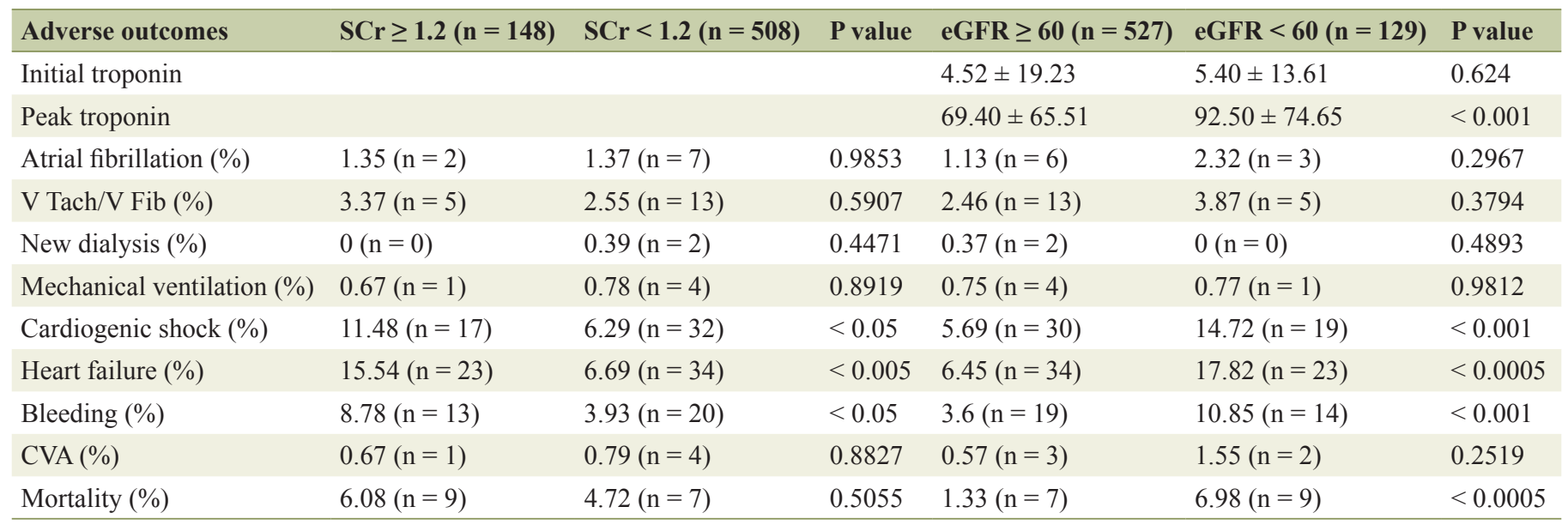

SCr: serum creatinine at admission; V Tach: ventricular tachycardia; V Fib: ventricular fibrillation; CVA: cerebrovascular accident; eGFR: estimated glomerular filtration rate at admission.

MI who subsequently underwent primary PCI, were included in the study. The electrocardiograms (EKGs) were reviewed by experienced cardiologists at CCAG. All coronary angiographies and primary PCIs were performed via the femoral and radial approach. Certain medications, e.g. aspirin, clopidogrel, rosuvastatin or atorvastatin, angiotensin converting enzyme inhibitor or angiotensin receptor blocker and betablockers were administered as per the standard guidelines and according to the hemodynamic status of the patients. Glycoprotein IIb/IIIa receptor blockers (e.g. tirofiban) were also used based on visualized thrombus burden. Patients with abnormal $\mathrm{SCr}$ at admission ( $\geq 1.2 \mathrm{mg} / \mathrm{dL}$ ) were generally older (mean age $66.84 \pm 13.66$ vs. $59.45 \pm 11.70, \mathrm{P} \leq 0.0001$ ). A significantly high percentage of the study population had prior history of hypertension $(\mathrm{P} \leq 0.005)$, dyslipidemia $(\mathrm{P} \leq$ $0.0005)$, myocardial infarction $(\mathrm{P}<0.005)$, heart failure $(\mathrm{P} \leq$ $0.05)$ and/or cerebrovascular diseases $(\mathrm{P} \leq 0.0005)$. Interestingly there was lesser number of patients with smoking history in abnormal SCr group (35.81\% vs. $52.75 \%, \mathrm{P} \leq 0.0005)$. Patients with eGFR CKD-EPI stage III $(<60 \mathrm{~mL} / \mathrm{min} / 1.73$ $\mathrm{m}^{2}$ ) on admission had an increased incidence of cardiogenic shock $(\mathrm{P}=0.001)$, bleeding $(\mathrm{P}<0.001)$, heart failure $(\mathrm{P}<$ $0.0005)$ and higher mortality rates $(\mathrm{P}=0.0005)$ (Tables 1 , 2). Furthermore, in the setting of STEMI, elevated SCr was associated with an increased risk of developing major adverse events like cardiogenic shock $(\mathrm{P}=0.05)$, bleeding $(\mathrm{P}=$ $0.05)$, heart failure $(\mathrm{P}=0.005)$. Patients with eGFR stage III $\left(<60 \mathrm{~mL} / \mathrm{min} / 1.73 \mathrm{~m}^{2}\right)$ had higher levels of cardiac enzymes (mean peak troponin level $92.50 \pm 74.65$ vs. $69.40 \pm 65.51$, $\mathrm{P}=0.001)$. Of note, there was no significant mortality difference between abnormal and normal SCr groups $(\mathrm{P}=0.5055)$. In addition, there was no statistically significant difference in the occurrence of adverse events like atrial fibrillation $(\mathrm{P}=$ $0.985, \mathrm{P}=0.296$ ), ventricular tachycardia/ventricular fibrillation $(\mathrm{P}=0.590, \mathrm{P}=0.379)$ and cerebrovascular accidents (CVAs) $(\mathrm{P}=0.882, \mathrm{P}=0.251)$ between abnormal $\mathrm{SCr}$ and lower eGFR $\left(<60 \mathrm{~mL} / \mathrm{min} / 1.73 \mathrm{~m}^{2}\right)$ groups compared to nor$\mathrm{mal} \mathrm{SCr}(<1.2 \mathrm{mg} / \mathrm{dL})$ and higher eGFR $(\geq 60 \mathrm{~mL} / \mathrm{min} / 1.73$ $\mathrm{m}^{2}$ ) groups respectively.

\section{Discussion}

Chronic kidney disease (CKD) is associated with arrhythmias, cardiomyopathy (dilated and hypertrophic variant), left ventricular (LV) hypertrophy and dysfunction, increased carotid intima-medial thickness (IMT), endothelial dysfunction (as measured by flow mediated dilation of the brachial artery), and calcification of the coronaries. These constitute independent predictors of cardiac morbidity and mortality [6]. CKD and uremia-induced decreased shortening at the myocardial midwall result in low contractile reserve during stress. This causes reduced global and segmental myocardial blood flow, leading to myocardial stunning [6].

In this study, patients with STEMI who had elevated admission $\mathrm{SCr}$ and eGFR $<60 \mathrm{~mL} / \mathrm{min} / 1.73 \mathrm{~m}^{2}$ showed an increased risk of developing major adverse clinical events including cardiogenic shock, bleeding and heart failure. In addition, mortality rates were significantly higher in patients with eGFR $<60 \mathrm{~mL} / \mathrm{min} / 1.73 \mathrm{~m}^{2}$. Cardiovascular morbidity and mortality is evident in early renal disease and in patients with CKD because of hemodynamic overload and several metabolic and endocrine imbalances that are fairly specific to uremia [7]. Furthermore, renal failure is associated with a procoagulant state, higher homocysteine levels, and endothelial dysfunction that promote atherosclerosis and plaque formation $[8,9]$. CKD with NSTEMI is associated with increased risk of in-hospital adverse events, which are complicated with longer hospital stay and higher medical costs [4]. In another study, longer hospital stay was observed in the lowest eGFR group due to increased in-hospital adverse events [10]. Studies have also shown that the risk of stroke increases with renal dysfunction $[10,11]$. However, our study did not show any difference in cerebrovascular accident rates between patients with normal and abnormal renal functions.

Regarding the classification of CKD, CKD-EPI correlated 
with MDRD and Cockcroft and Gault (CG) formula 92\% of the time [12]. All three definitions correlate well with outcomes; however CKD-EPI predicts worse prognosis better than MDRD or CG in STEMI patients [12]. The limitations of calculating renal functions from SCr alone or from a $24 \mathrm{~h}$ urine creatinine clearance, and the cost and complexities involved in measuring endogenous eGFR or determining inulin/ radionuclide based gold standard GFR lead to eGFR calculation [12]. Authors feel the CKD-EPI equation includes log SCr (modeled as a two-slope linear spline with sex specific knots at $62 \mu \mathrm{mol} / \mathrm{L}$ in women and $80 \mu \mathrm{mol} / \mathrm{L}$ in men), with gender, race and age on the natural scale. It is therefore effectively four different equations for Caucasians (men, women, above the knot value, below the knot value) and another four for African-Americans in whom a different factor is used considerably outweighing their reliability and making them unsuitable for routine use in the patients with STEMI [12]. Regardless of definition, increased admission $\mathrm{SCr}$ corresponded with increased risk of major adverse cardiovascular outcomes among STEMI patients undergoing PCI. However, in our study, except for cardiogenic shock, bleeding and heart failure, there was no significant difference in the occurrence of atrial fibrillation, ventricular tachycardia/ventricular fibrillation and CVAs between elevated $\mathrm{SCr}$ and lower eGFR $(<60 \mathrm{~mL} / \mathrm{min} / 1.73$ $\left.\mathrm{m}^{2}\right)$ groups compared to normal $\mathrm{SCr}(<1.2 \mathrm{mg} / \mathrm{dL})$ and higher eGFR ( $\geq 60 \mathrm{~mL} / \mathrm{min} / 1.73 \mathrm{~m}^{2}$ ) groups respectively.

Our study also showed higher percentage of patients with common comorbidities (hypertension, diabetes, and dyslipidemia) and prior history of myocardial infarction, heart failure and cerebrovascular disease among elevated $\mathrm{SCr}$ group. These patients were also commonly male with an older age. These clinical factors are associated with poor prognosis in the setting of acute myocardial infarction. Multivariate regression analysis showed mild deterioration of eGFR (derived per CKD-EPI) was prognostic in STEMI patients undergoing primary PCI. Our cohort with CKD stage III or greater was associated with higher rates of decreased left ventricular function, higher troponins levels and increased mortality rates. This study was in accordance with Sonmez et al who showed that an eGFR $<60$ $\mathrm{mL} / \mathrm{min} / 1.73 \mathrm{~m}^{2}$ was associated with reduced left ventricular ejection fraction and larger myocardial infarct size in patients undergoing primary PCI for STEMI [13].

Use of a standardized, evidence-based approach involving careful evaluation of GFR on admission for STEMI patients undergoing PCI is essential. Early recognition of patients at risk for development of $\mathrm{AKI} / \mathrm{CKD}$ post-PCI is paramount to improve the outcomes of an otherwise life-saving therapy. These findings emphasize the importance of a multidisciplinary approach involving nephrologists and interventionists for early recognition and management of AKI/CKD in patients with STEMI undergoing PCI. Future large-scale prospective studies are needed to confirm our findings. Additionally, CKD-EPI derived eGFR should be used for prediction of in-hospital and long-term mortality in STEMI patients undergoing primary PCI.

\section{Conclusions}

In the setting of STEMI, elevated $\mathrm{SCr}$ and eGFR $<60 \mathrm{~mL} /$ $\min / 1.73 \mathrm{~m}^{2}$ were associated with an increased risk of developing major adverse clinical events including cardiogenic shock, bleeding and heart failure in patients undergoing PCI. In-hospital adverse events are complicated by longer hospital stay and higher medical costs; therefore it is imperative to identify those at greater risk earlier in their clinical course. Our findings need to be validated in a larger group and multi-center registry for a better understanding of eGFR and adverse outcomes after PCI following STEMI.

\section{Conflict of Interest}

The authors have no conflict of interest to declare

\section{Funding}

This research received no specific grant from any funding agency in the public, commercial, or not-for-profit sectors.

\section{References}

1. Kim JY, Jeong MH, Ahn YK, Moon JH, Chae SC, Hur $\mathrm{SH}$, Hong TJ, et al. Decreased glomerular filtration rate is an independent predictor of in-hospital mortality in patients with ST-segment elevation myocardial infarction undergoing primary percutaneous coronary intervention. Korean Circ J. 2011;41(4):184-190.

2. Akkaya E, Ayhan E, Uyarel H, Ergelen M, Turer A, Demirci D, Demirci D, et al. [The impact of chronic kidney disease on in-hospital clinical outcomes in patients undergoing primary percutaneous angioplasty for STsegment elevation myocardial infarction]. Turk Kardiyol Dern Ars. 2011;39(4):276-282.

3. Anavekar NS, McMurray JJ, Velazquez EJ, Solomon SD, Kober L, Rouleau JL, White HD, et al. Relation between renal dysfunction and cardiovascular outcomes after myocardial infarction. N Engl J Med. 2004;351(13):12851295.

4. Uluganyan M, Karaca G, Ulutas TK, Ekmekci A, Tusun E, Murat A, Koroglu B, et al. The impact of admission serum creatinine derived estimated glomerular filtration rate on major adverse cardiac events in ST-segment elevation myocardial infarction patients undergoing primary percutaneous coronary intervention. J Clin Med Res. 2016;8(4):325-330.

5. Estimating glomerular filtration rate - The national institute of diabetes and digestive and kidney diseases. Available from: https://www.niddk.nih.gov/health-information/ communication-programs/nkdep/laboratory-evaluation/ glomerular-filtration-rate/estimating.

6. Zhao L, Wang L, Zhang Y. Elevated admission serum creatinine predicts poor myocardial blood flow and one-year mortality in ST-segment elevation myocardial infarction patients undergoing primary percutaneous coronary intervention. J Invasive Cardiol. 2009;21(10):493-498. 
7. Seyfarth M, Kastrati A, Mann JF, Ndrepepa G, Byrne RA, Schulz S, Mehilli J, et al. Prognostic value of kidney function in patients with ST-elevation and nonST-elevation acute myocardial infarction treated with percutaneous coronary intervention. Am J Kidney Dis. 2009;54(5):830-839.

8. Shacham Y, Leshem-Rubinow E, Gal-Oz A, Ben-Assa E, Steinvil A, Keren G, Roth A, et al. Relation of in-hospital serum creatinine change patterns and outcomes among ST-segment elevation myocardial infarction patients undergoing primary percutaneous coronary intervention. Clin Cardiol. 2015;38(5):274-279.

9. Chronic Kidney Disease Prognosis Consortium, Matsushita K, van der Velde M, Astor BC, Woodward M, Levey AS, de Jong PE, et al. Association of estimated glomerular filtration rate and albuminuria with all-cause and cardiovascular mortality in general population cohorts: a collaborative meta-analysis. Lancet. 2010;375(9731):20732081.
10. AlFaleh HF, Alsuwaida AO, Ullah A, Hersi A, AlHabib KF, AlShahrani A, AlNemer K, et al. Glomerular filtration rate estimated by the CKD-EPI formula is a powerful predictor of in-hospital adverse clinical outcomes after an acute coronary syndrome. Angiology. 2012;63(2):119126.

11. Wannamethee SG, Shaper AG, Perry IJ. Serum creatinine concentration and risk of cardiovascular disease: a possible marker for increased risk of stroke. Stroke. 1997;28(3):557-563.

12. Schold JD, Navaneethan SD, Jolly SE, Poggio ED, Arrigain S, Saupe W, Jain A, et al. Implications of the CKD-EPI GFR estimation equation in clinical practice. Clin J Am Soc Nephrol. 2011;6(3):497-504.

13. Sonmez O, Vatankulu MA, Tasal A, Bacaksiz A, Ayhan S, Yazici HU, Karakaya E, et al. Does estimated glomerular filtration rate have an effect on left ventricular function after ST-elevation myocardial infarction? Echocardiography. 2014;31(3):318-324. 